\title{
Optical alignment of the Chromospheric Lyman-Alpha SpectroPolarimeter using sophisticated methods to minimize activities under vacuum.
}

\author{
G. Giono $^{a}$, Y. Katsukawa ${ }^{a}$, R.Ishikawa ${ }^{a}$, N. Narukage $^{a}$, R. Kano $^{a}$, M. Kubo $^{a}$, S. Ishikawa $^{b, c}$, \\ T. Bando ${ }^{a}$, H. Hara ${ }^{a}$, Y. Suematsu ${ }^{a}$, A. Winebarger ${ }^{d}$, K. Kobayashi ${ }^{d}$, F. Auchère ${ }^{e}$ and J. \\ Trujillo Bueno $f$ \\ ${ }^{a}$ National Astronomical Observatory of Japan, Mitaka, JAPAN \\ ${ }^{b}$ Japan Aerospace Exploration Agency, Tokyo, JAPAN \\ ${ }^{c}$ Institute of Space and Astronautical Science, Tokyo, JAPAN \\ ${ }^{d}$ Marshall Space Flight Center, Huntsville, USA \\ eInstitut d'Astrophysique Spatiale, Orsay, FRANCE \\ Instituto de Astrofisica de Canarias, La Laguna, SPAIN
}

\begin{abstract}
The Chromospheric Lyman-Alpha Spectro-Polarimeter (CLASP) is a sounding-rocket instrument developed at the National Astronomical Observatory of Japan (NAOJ) as a part of an international collaboration. The instrument main scientific goal is to achieve polarization measurement of the Lyman- $\alpha$ line at $121.56 \mathrm{~nm}$ emitted from the solar upper-chromosphere and transition region with an unprecedented $0.1 \%$ accuracy. For this purpose, the optics are composed of a Cassegrain telescope coated with a "cold mirror" coating optimized for UV reflection and a dual-channel spectrograph allowing for simultaneous observation of the two orthogonal states of polarization. Although the polarization sensitivity is the most important aspect of the instrument, the spatial and spectral resolutions of the instrument are also crucial to observe the chromospheric features and resolve the Ly- $\alpha$ profiles. A precise alignment of the optics is required to ensure the resolutions, but experiments under vacuum conditions are needed since Ly- $\alpha$ is absorbed by air, making the alignment experiments difficult. To bypass this issue, we developed methods to align the telescope and the spectrograph separately in visible light. We will explain these methods and present the results for the optical alignment of the CLASP telescope and spectrograph. We will then discuss the combined performances of both parts to derive the expected resolutions of the instrument, and compare them with the flight observations performed on September $3^{\text {rd }} 2015$.
\end{abstract}

Keywords: Sun, CLASP, Lyman-alpha, Alignment

\section{INTRODUCTION}

The Chromospheric Lyman-alpha SpectroPolarimeter $\left(\mathrm{CLASP}^{1,2}\right)$ is a sounding rocket experiment developed at the National Astronomical Observatory of Japan as part of an international collaboration including the NASA Marshall Space Flight Center in USA, the Institut d'Astrophysique Spatiale in France and the Instituto de Astrofísica de Canarias in Spain. The main scientific goal of the instrument is to achieve the first polarization measurement of the Lyman- $\alpha$ line $(121.56 \mathrm{~nm})$ which is emitted in the solar upper-chromosphere and transition region. This polarization measurement can be used to detect the Hanle effect signature (i.e. modification of the linear polarization produced by scattering processes due to the presence of magnetic field) and therefore determine the magnetic field strength and orientation in these atmospheric layers. ${ }^{3,4}$ Understanding these properties of the magnetic field is crucial to address some of the major issues in solar physics, such as the coronal heating, and to confront our theoretical models of the solar atmosphere. These models have given hints about the nature of the chromospheric magnetic field, but have yet to be confirmed with real and direct observations.

Send correspondence to G.Giono at gabriel.giono@nao.ac.jp 
Table 1. RMS spot radius requirement and corresponding resolution at the edge of the slit for the telescope and spectrograph. The combined performances are calculated as the root sum square of both parts, also considering the 0.93 magnification of the spectrograph.

\begin{tabular}{|c|c|c|c|c|}
\hline & $\begin{array}{c}\text { Spatial RMS } \\
\text { spot radius }\end{array}$ & $\begin{array}{c}\text { Spatial } \\
\text { resolution }\end{array}$ & $\begin{array}{c}\text { Spectral RMS } \\
\text { spot radius }\end{array}$ & $\begin{array}{c}\text { Spectral } \\
\text { resolution }\end{array}$ \\
\hline Telescope & $13.0 \mu \mathrm{m}$ & $2.1^{\prime \prime}$ & - & - \\
Spectrograph & $13.5 \mu \mathrm{m}$ & $2.3^{\prime \prime}$ & $13.5 \mu \mathrm{m}$ & $0.010 \mathrm{~nm}$ \\
Combined & $18.1 \mu \mathrm{m}$ & $3.1^{\prime \prime}$ & $13.5 \mu \mathrm{m}$ & $0.010 \mathrm{~nm}$ \\
\hline
\end{tabular}

To achieve its scientific requirements, CLASP's optics are composed of a telescope and a spectrograph (see Figure 1). The particularity of the classical two-mirrors Cassegrain telescope lays in its optimization for UVreflection: the primary mirror is coated with a narrow-band filtering coating (i.e. "cold mirror" coating) providing a reflectivity at Ly- $\alpha$ close to $55 \%$ while transmitting visible-light up to $96.5 \%$ and the secondary mirror received an Aluminium coating enhanced with $\mathrm{MgF}_{2}$, also to improve the UV reflection. Similarly, the design of the spectrograph was selected to minimize the number of optical surfaces in order to improve the throughput and therefore the polarization accuracy, with its dual-channel allowing for simultaneous measurements of the two orthogonal polarization states. The combined performance of both parts of the instrument are designed to achieve a 3.1" spatial resolution and a $0.010 \mathrm{~nm}$ spectral resolution at the edge of the slit. These requirements in terms of the corresponding RMS spot radius are shown in Figure 1. Although achieving the polarization accuracy is crucial for detecting the Hanle effect, reaching the required spatial and spectral resolutions is also extremely important in order to properly resolve chromospheric structures (e.g. thread-like structures observed by the VAULT Ly- $\alpha$ sounding rocket ${ }^{5}$ ) and to ensure a well-resolved Ly- $\alpha$ profile. Therefore, a careful alignment of all the optical elements is needed but raised a complicated technical challenge. Indeed, the Ly- $\alpha$ wavelength is absorbed by air: the alignment has to be performed under vacuum conditions which makes any experiment difficult. To pass-by this issue, we developed and tested a custom alignment procedure ${ }^{6}$ with sophisticated methods to align the telescope and spectrograph separately almost entirely in visible-light.

In the following, we are going recall the alignment procedure and present the results of the optical alignment performed during the summer 2015 for both the telescope and the spectrograph. Then, we will discuss the combined performances of the instrument and compare these results with the required tolerance. Finally, the resolutions achieved during the flight of the instrument on September $3^{\text {rd }} 2015$ will be compared with the expected pre-flight performances, and we will conclude on the success of the optical alignment.

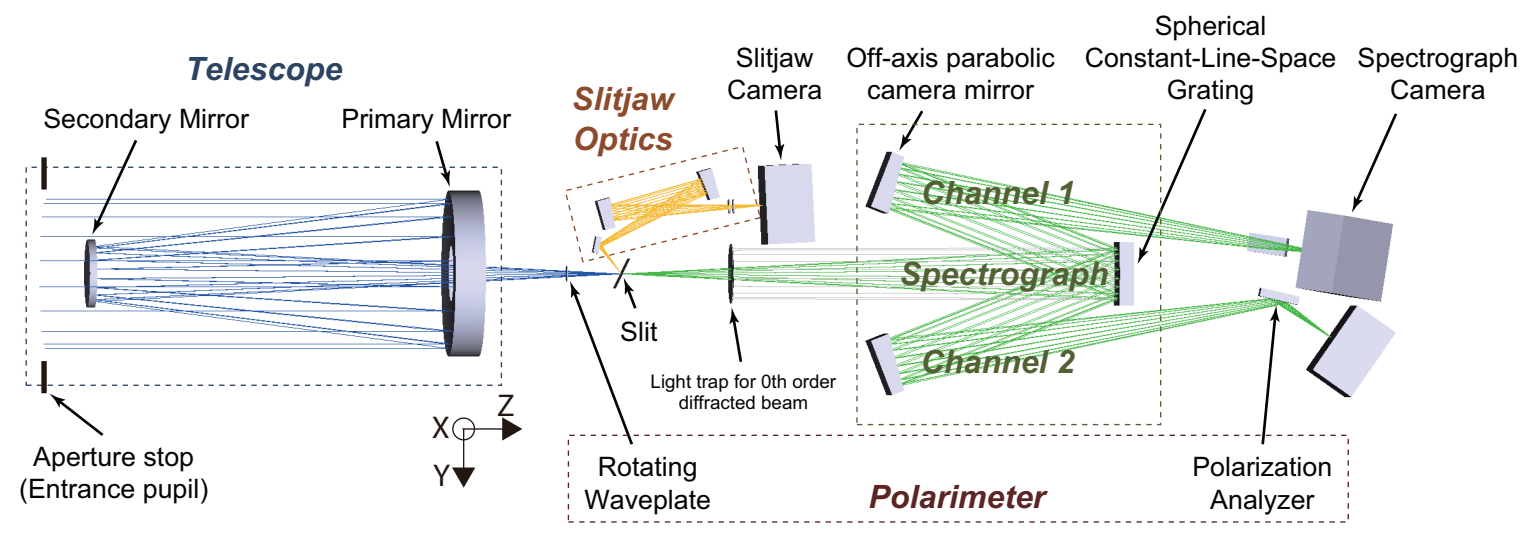

Figure 1. CLASP instrument design ${ }^{7}$ 


\section{OPTICAL ALIGNMENT OF THE TELESCOPE}

The telescope part of the CLASP instrument is a two-mirror $\phi 270 \mathrm{~mm}$ entrance aperture Cassegrain telescope with a F/9.68 number and a $2614 \mathrm{~mm}$ focal length (more details ${ }^{6}$ ), focusing the light onto a 400" length and 1.45 " width slit. The purpose of the alignment is to align the secondary mirror tilts (around the X-axis and the $\mathrm{Y}$-axis) and despace (along the Z-axis) with respect to the primary mirror in order to remove coma and defocus aberrations at the center of the slit. Because the reflection by the mirrors is not wavelength dependent, the alignment was conducted in visible-light instead of Ly- $\alpha$. A He-Ne laser interferometer (632.8 nm) was used in a double-pass configuration ${ }^{6}$ to measure the telescope wavefront error (WFE): the interferometer was precisely adjusted orthogonally to the slit position using a 6-axis table and a mirror-target located at the slit position (i.e. mirror with concentric coated and uncoated rings). The beam was reflected by the primary mirror, the secondary mirror and exited by the telescope's entrance aperture. Then, a large flat mirror ( $\phi 600 \mathrm{~mm}, \mathrm{RMS}$ WFE $\left.15 \mathrm{~nm}^{8}\right)$ positioned in front of the telescope reflected the beam back to the telescope and then to the interferometer to get interference fringes. The WFE was retrieved from the measured interference fringes using a phase-carrier method. ${ }^{9}$ However, due to the primary mirror's "cold mirror" coating, only less than $0.1 \%$ of the light was reflected for the interference. Therefore, extensive measurements were performed to accurately estimate the WFEs, each of which were obtained by averaging the results from twenty fringe maps. The optical aberrations were extracted from the WFE by fitting the first 37th Zernike polynomials, and optical simulations were used to derive the relationship between the misalignment of the secondary mirror and the amplitude of the optical aberrations (i.e. Zernike coefficient, in wave). These relationships between the coma/tilt and defocus/despace were used to derive the amount of correction required on the secondary mirror and implemented by shimming at the base of the mirror's holder.

Multiple measurements and iterations were conducted to remove the coma and defocus aberrations at the center of the field of view. The final WFE after the alignment is presented in the left part of Figure 2, measured in zero-G condition by averaging three measurements taken with the telescope rotated along the Z-axis by $120^{\circ}$ each (due to the three-points attachment of the telescope structure) to cancel the effect of gravity onto the WFE. Table 2 presents the corresponding aberration coefficients derived from the Zernike polynomials fitting. These coefficients are given in unit of $\lambda$ (i.e. wave, with $\lambda=632.8 \mathrm{~nm}$ ), and the error was estimated using the standard deviation on the aberration coefficient from the twenty WFEs (i.e. derived from each of the twenty fringe maps) composing each WFE measurement. As shown by Table 2, the coma aberrations were successfully reduced to a comparable level as the achievable accuracy. The telescope Point Spread Function (PSF) was computed using a ray-tracing software, by including the zero-G WFE measurement into the optical model of the telescope. This PSF (presented in the right part of Figure 2) includes the diffraction effect from the entrance aperture at the Ly- $\alpha$ wavelength and its RMS spot radius was estimated at $7.7 \mu \mathrm{m}$.

Table 2. Aberration coefficients (Zernike coefficients) in unit of $\lambda=632.8 \mathrm{~nm}$ for the zero-G WFE. Error is given as the standard deviation out of the aberration coefficient of the twenty WFEs measurements composing each WFE measurement.

\begin{tabular}{|c|c|c|c|c|c|}
\hline Aberrations & Defocus & Astigmatism $0^{\circ}$ & Astigmatism 45 & Coma X & Coma Y \\
\hline Zernike coefficient $(\lambda)$ & 0.18 & -0.07 & -0.08 & 0.02 & -0.01 \\
\hline Error $(\lambda)$ & \pm 0.10 & \pm 0.04 & \pm 0.04 & \pm 0.03 & \pm 0.03 \\
\hline
\end{tabular}

Since the $13.0 \mu \mathrm{m}$ requirement for RMS spot radius was defined at the edge of the slit, additional WFE measurements were performed across the field of view by adjusting the position of the laser interferometer with respect to the slit plane (i.e. mirror target surface) using the motorized 6-axis table. The RMS WFE and corresponding RMS spot radius for these measurements are shown in Table 3. These measurements were performed at the top and bottom of the slit (i.e. $X \pm 200 "$ "), as well as perpendicularly to the slit (i.e. $Y$ $\pm 200 ")$, and were recorded for a fixed orientation of the telescope. The coefficient of the astigmatism aberrations created by gravity for this particular orientation of the telescope was estimated from the zero-G measurements and removed from these measurements as an approximated correction for gravity effect. Results from Table 3 confirmed that the $13.0 \mathrm{\mu m}$ RMS spot radius requirement for the telescope was achieved at the edge of the slit. 

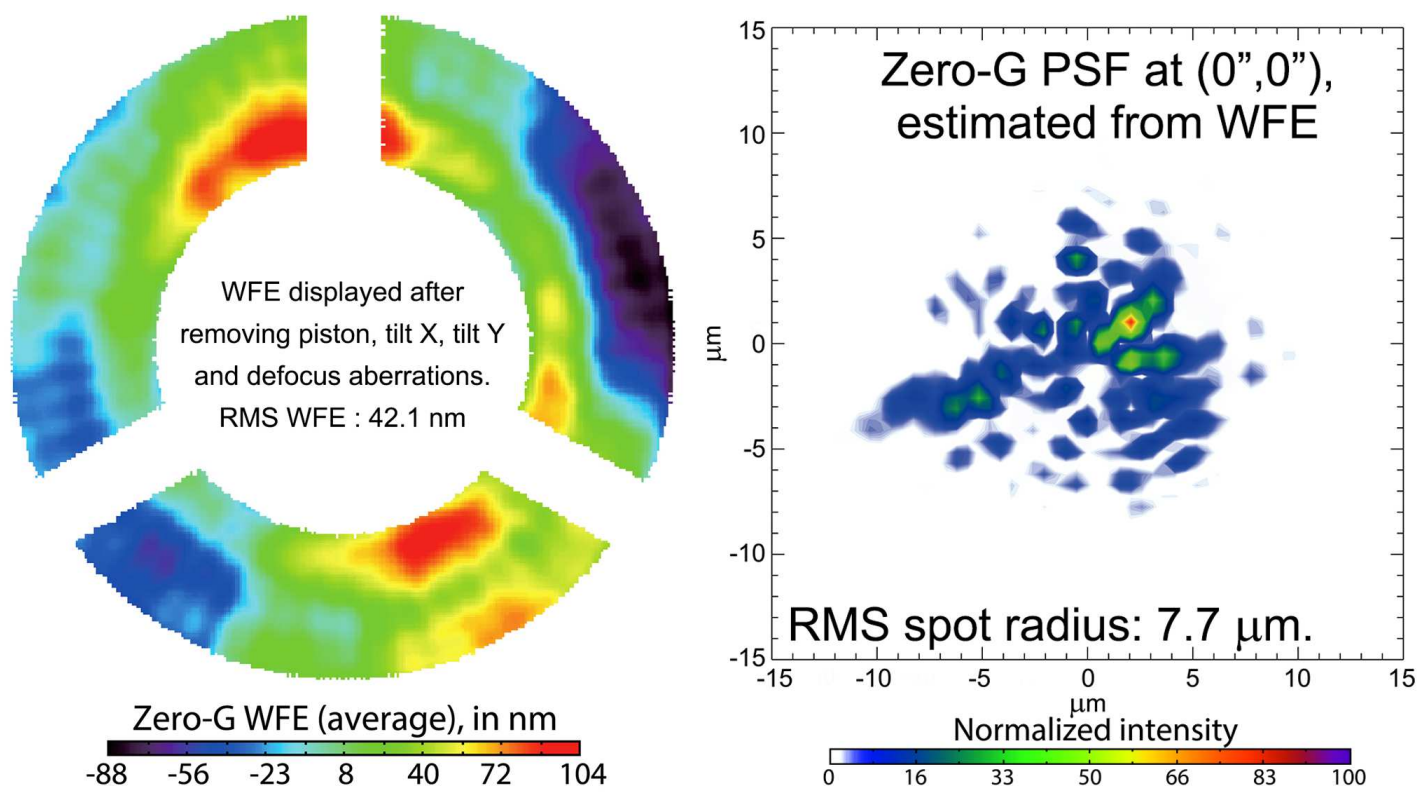

Figure 2. Left: Final zero-G WFE after alignment of the secondary mirror. Piston, tilts and defocus aberrations were removed. RMS WFE: $42.1 \mathrm{~nm}$. Right: Corresponding PSF at Ly- $\alpha$. RMS spot radius: $7.7 \mathrm{\mu m}$.

Table 3. RMS WFE and corresponding RMS spot radius for the measurements taken across the field of view.

\begin{tabular}{|c|c|c|c|c|c|}
\hline Position $(\mathrm{X}, \mathrm{Y})$ & $(0 ", 0 ")$ & $(+200 ", 0 ")$ & $(-200 ", 0 ")$ & $(+200 ", 0 ")$ & $(-200 ", 0 ")$ \\
\hline RMS WFE & $40.0 \mathrm{~nm}$ & $39.7 \mathrm{~nm}$ & $36.7 \mathrm{~nm}$ & $45.6 \mathrm{~nm}$ & $30.3 \mathrm{~nm}$ \\
\hline RMS spot radius & $7.7 \mu \mathrm{m}$ & $7.8 \mu \mathrm{m}$ & $8.1 \mu \mathrm{m}$ & $8.5 \mu \mathrm{m}$ & $7.8 \mu \mathrm{m}$ \\
\hline
\end{tabular}

Note that the defocus aberration was not completely removed from the WFE at the center of the slit. The final focus adjustment was performed when the telescope was attached to the spectrograph: a $\pm 0.5 \mathrm{~mm}$ stroke was allocated for the adjustment between both the parts. This stoke roughly corresponds to a $\pm 0.5 \lambda$ change in the defocus aberration coefficient, and allowed to remove the remaining defocus aberration. The optical simulations were also used to estimate the change of the RMS spot radius at the edge of the slit when considering the \pm 0.1 $\mathrm{mm}$ tolerance allocated for this focus adjustment. The resulting $10.8 \mu \mathrm{m}$ RMS spot radius for the worst case scenario was still within the $13.0 \mu \mathrm{m}$ requirement. In conclusion, the optical alignment of the telescope was successfully conducted in visible-light despite the primary mirror's "cold mirror" coating, and achieved the 13.0 $\mu \mathrm{m}$ RMS spot radius requirement at the edge of the slit.

\section{OPTICAL ALIGNMENT OF THE SPECTROGRAPH}

Six degrees of freedom have to be adjusted for the alignment of the spectrograph in order to obtain the required image quality: the grating's X, Y and Z tilts, the off-axis mirror's (M3) X and Y tilts and the CCD camera focus position along Z-axis. Because not every optical surfaces had to be align at the Ly- $\alpha$ wavelength, a custom alignment procedure minimizing the activities under vacuum condition was previously presented and tested. ${ }^{6}$ In this procedure, the M3 tilts mirrors are aligned using a He-Ne laser by replacing the flight Ly- $\alpha$ grating with a custom-made alignment grating. This alignment grating was specifically designed with the same specifications (clear aperture, curvature radius and conic constant) as the flight grating, but with a ruling density selected to provide the same diffraction angle at the He-Ne wavelength as the flight grating in Ly- $\alpha$. At first, the X-tilt and Y-tilt of the grating were aligned to the spectrograph's mechanical axis (represented with a mirror-target) using a theodolite. Then, the alignment grating Z-tilt was adjusted in two steps: the slit was illuminated using the monochromatic He-Ne laser to provide the slit direction on the images taken by the $4.4 \mu \mathrm{m}$ pixel CCD cameras 
used for the alignment in visible-light. The slit was then replaced by a pinhole array (five $\phi 10 \mu \mathrm{m}$ pinholes along the slit direction, at -200 ", $-100 ", 0$ ", +100 " and +200 ") and illuminated using white-light to observe the dispersion direction on the CCDs. Because the grating Z-tilt changes the orientation of the ruling direction thus changing the dispersion direction with respect to the slit direction, this degree of freedom was adjusted to get the dispersion direction perpendicular to the slit direction, as observed by the CCD detectors. Finally, the misalignment on the M3's X-tilt and Y-tilt were estimated by illuminating the pinhole array with the He-Ne laser and by measuring the shape of the PSF (i.e. pinhole images). Optical simulations were conducted using a ray-tracing software to estimate the dependency of the PSF along the slit with respect to the X-tilt and Y-tilt of the off-axis mirrors. These simulations were used as a comparison with the measured PSF to diagnose the mirror's misalignments and to derive the amount of correction required by shimming. This alignment procedure in visible-light was successfully conducted, and the final spots are shown in Figure 3 for both the channels. The RMS spot radius at the edge of the slit fulfilled the $13.5 \mu \mathrm{m}$ requirements.

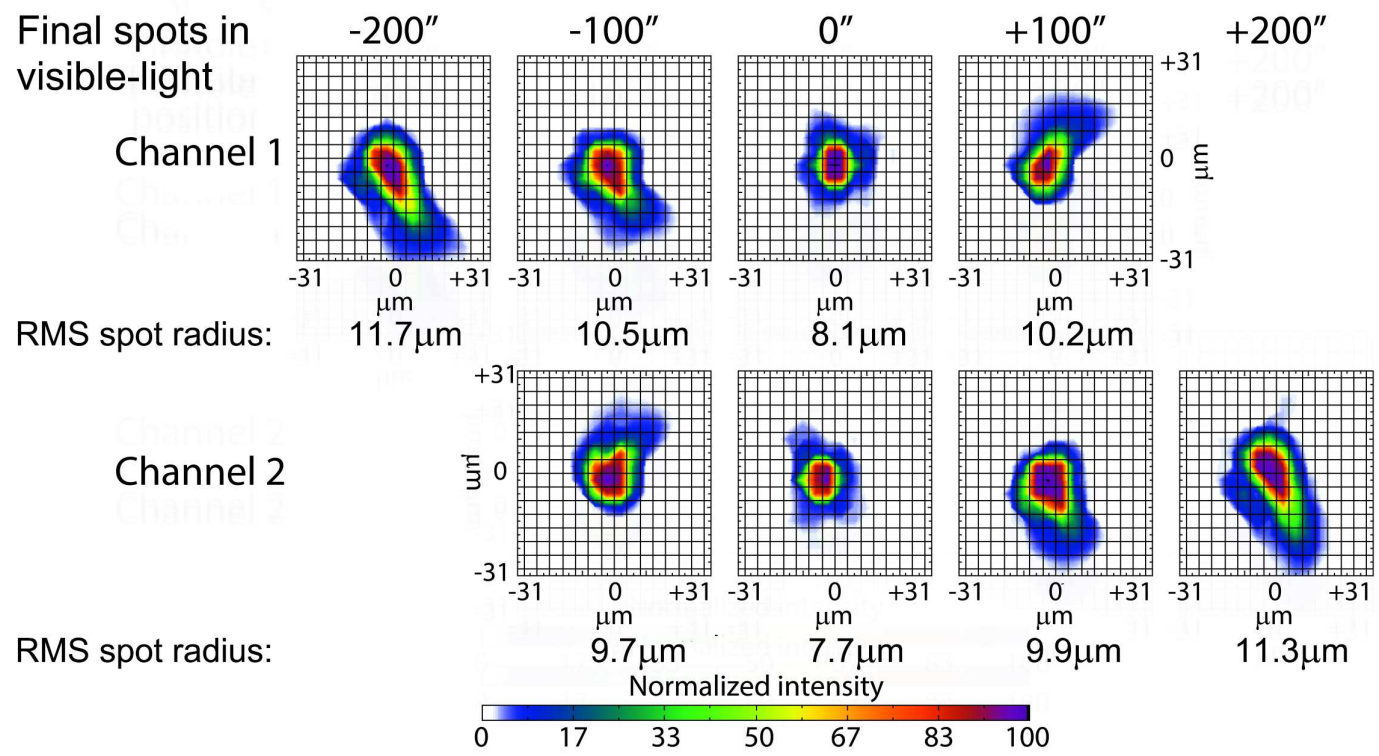

Figure 3. Final spots for both the channels after the alignment in visible-light for the five pinholes along the slit. The grid shows the $4.4 \mathrm{\mu m}$ CCD pixels.

With the M3s aligned, the alignment grating was replaced by the flight Ly- $\alpha$ grating and the CCD cameras used for the alignment in visible-light were replaced by the flight CCD cameras (13.0 $\mu \mathrm{m}$ pixel). The Xtilt and Y-tilt of the flight grating was also aligned with respect to the mechanical-axis using a theodolite. Then, only its Z-tilt, as well as the CCD focus position along the Z-axis, were adjusted under vacuum using motorized adjustment stages. These motorized adjustment stages were not intended to fly with the instrument and were therefore removed after the alignment. For the CCD cameras, the distance between the cameras and the spectrograph's structure was measured after the adjustment of their focus position. Based on this measurement, a custom shim plate was manufactured in order to position each camera at its focus location after removing the motorized adjustment stages. On the other hand, the grating-Z tilt was fixed after its adjustment with a metal plate attaching its rotating stage to the spectrograph's structure before removing the motorized adjustment mechanism. A deuterium lamp was used to illuminate the pinhole array and provided strong emissions at the deuterium and hydrogen Ly- $\alpha$ wavelengths (121.534 $\mathrm{nm}$ and $121.567 \mathrm{~nm}$, respectively). However, these two emission lines were intrinsically broad, making an estimation of the RMS spot radius inaccurate: a weaker emission line at $121.709 \mathrm{~nm}$ was used instead. The final spots at this wavelength are shown in Figure 4 for both the channels at the center of the slit. Due to the broader "continuum" in the wavelength direction, a Gaussian fitting was used in the spatial and spectral directions to estimate the RMS spot radius in these two directions instead of using the strait-forward calculation. Indeed, the RMS spot radius is equal to the Gaussian $\sigma$ for a one-dimensional distribution, assuming the spot has a Gaussian shape. This assumption was acceptable in 

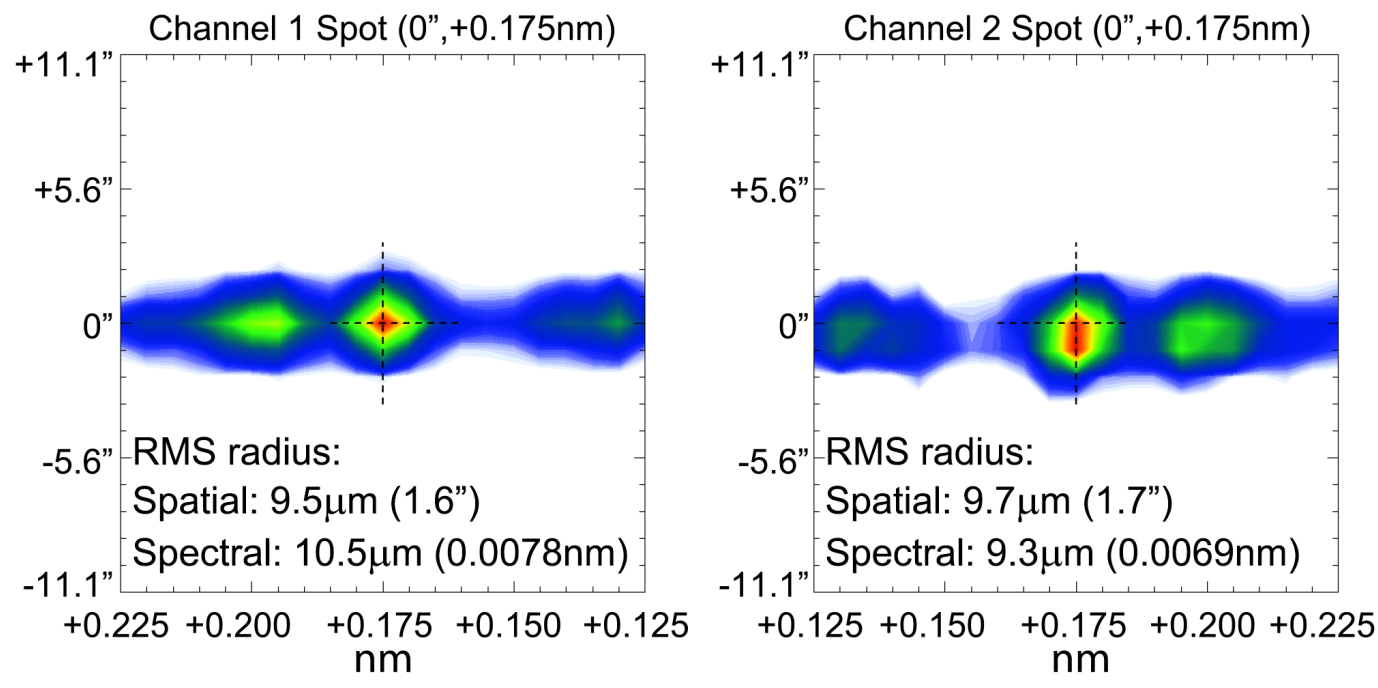

Figure 4. Final spot after the alignment under vaccum for both the channels at $121.709 \mathrm{~nm}$.

Table 4. Final RMS spot radius estimated at the edge of the slit using the measurement shown in Figure 4 and the ratio edge/center from Figure 3.

\begin{tabular}{|c|c|c|c|c|}
\hline & $\begin{array}{c}\text { Channel 1 } \\
\text { Spatial }\end{array}$ & $\begin{array}{c}\text { Channel 2 } \\
\text { Spatial }\end{array}$ & $\begin{array}{c}\text { Channel 1 } \\
\text { Spectral }\end{array}$ & $\begin{array}{c}\text { Channel 2 } \\
\text { Spectral }\end{array}$ \\
\hline Measured RMS spot radius at 0" & $9.5 \mu \mathrm{m}$ & $9.7 \mu \mathrm{m}$ & $10.5 \mu \mathrm{m}$ & $9.3 \mu \mathrm{m}$ \\
\hline Ratio edge/center (Figure 3) & 1.44 & 1.47 & 1.44 & 1.47 \\
\hline Estimated RMS spot radius at $\pm 200 "$ & $13.7 \mu \mathrm{m}$ & $14.3 \mu \mathrm{m}$ & $15.1 \mu \mathrm{m}$ & $13.7 \mu \mathrm{m}$ \\
\hline RMS spot radius requirement at $\pm 200 "$ & $13.5 \mu \mathrm{m}$ & $13.5 \mu \mathrm{m}$ & $13.5 \mu \mathrm{m}$ & $13.5 \mu \mathrm{m}$ \\
\hline
\end{tabular}

the case of the pinhole image because the diffraction effect at $\mathrm{Ly}-\alpha$ are small. Unfortunately, the spots were only measured at the center of the slit due to time-constraint on the instrument development. Nevertheless, an estimation of the RMS spot radius at the edge of the slit was possible using the ratio edge/center measured during the alignment in visible-light. Results are presented in Table 4 and show a slightly larger RMS spot radius than the $13.5 \mathrm{\mu m}$ requirement. Reaching the required accuracy at the edge of the slit was expected to be difficult from design, and the estimation presented in Table 4 might also have been affected by the sampling size of the CCD cameras due to the $13.0 \mu \mathrm{m}$ pixel size. For these reasons, the estimated RMS spot radius was considered acceptable for the spectrograph. Hence, the spectrograph was successfully aligned using our custom procedure designed to minimize activities under vacuum.

\section{COMBINED PERFORMANCES}

The combined performance of the CLASP instrument were calculated as the root sum square of the telescope's and spectrograph's RMS spot radius at the edge of the slit shown in Section 2 and Section 3, taking into account the 0.93 magnification of the spectrograph. Results are shown in Table 5 and are compared to the requirement from Table 1. Note that the performance achieved for the telescope compensates for the larger spatial RMS spot radius measured for the spectrograph and ensured the spatial resolution in the two channels. On the other hand, the spectral resolution was slightly above the requirement at the edge of the slit for both the channels, as discussed in Section 3. 
Table 5. Combined performance of the instrument at the edge of the slit in term of RMS spot radius and resolution, compared to the requirement from Table 1.

\begin{tabular}{|c|c|c|c|c|}
\hline $\begin{array}{c}\text { RMS spot radius } \\
\text { (resolution) }\end{array}$ & $\begin{array}{c}\text { Channel 1 } \\
\text { Spatial resolution }\end{array}$ & $\begin{array}{c}\text { Channel 2 } \\
\text { Spatial resolution }\end{array}$ & $\begin{array}{c}\text { Channel 1 } \\
\text { Spectral resolution }\end{array}$ & $\begin{array}{c}\text { Channel 2 } \\
\text { Spectral resolution }\end{array}$ \\
\hline Telescope & $8.5 \mu \mathrm{m}\left(1.3^{\prime \prime}\right)$ & $8.5 \mu \mathrm{m}\left(1.3^{\prime \prime}\right)$ & - & - \\
Spectrograph & $13.7 \mu \mathrm{m}\left(2.3^{\prime \prime}\right)$ & $14.3 \mu \mathrm{m}\left(2.4^{\prime \prime}\right)$ & $15.2 \mu \mathrm{m}(0.0112 \mathrm{~nm})$ & $13.7 \mu \mathrm{m}(0.0101 \mathrm{~nm})$ \\
Combined & $15.8 \mu \mathrm{m}\left(2.7^{\prime \prime}\right)$ & $16.3 \mu \mathrm{m}\left(2.8^{\prime \prime}\right)$ & $15.2 \mu \mathrm{m}(0.0112 \mathrm{~nm})$ & $13.7 \mu \mathrm{m}(0.0101 \mathrm{~nm})$ \\
\hline Requirement & $18.1 \mu \mathrm{m}\left(3.1^{\prime \prime}\right)$ & $18.1 \mu \mathrm{m}\left(3.1^{\prime \prime}\right)$ & $13.5 \mu \mathrm{m}(0.0100 \mathrm{~nm})$ & $13.5 \mu \mathrm{m}(0.0100 \mathrm{~nm})$ \\
\hline
\end{tabular}

\section{COMPARISON WITH THE FLIGHT RESULTS}

CLASP was successfully launched on September $3^{r d} 2015$, and observed the Sun for about five minutes, both at the disc center $(\sim 15 \mathrm{~s})$ and close to the limb (i.e. main science target, $\sim 240 \mathrm{~s})$. The recorded images by the slit-jaw and spectrograph cameras were used to determine the achieved spatial and spectral resolutions.

For the spatial resolution, the estimation was performed by analysing the power spectrum of the slit-jaw's images and spectrograph's spectrums along the slit. Figure 5 shows the power spectrum (integrated radially) for three $160 \times 160$ pixels regions (i.e. $\sim 165 " \times 165 "$ ) located at the top, middle and bottom of the slit, extracted from a slit-jaw image taken at the center of the solar disc. The limiting frequency (i.e. where the power spectrum reach the power of the Nyquist frequency) seems to be around 2.5" at the top, middle and bottom of the slit. Note that this limiting frequency does not strictly indicates the limit of resolution because the power spectrum is actually a combination of the instrument's Modulation Transfer Function (MTF, characterizing the instrument's performances and resolution at its cut-off frequency) and of the frequencies contained in the observed image (i.e. the solar structures, including the frequencies from the noise). However, these solar structures can be assumed to be have smaller scales than the Nyquist resolution of 2.2" (i.e. two pixels), e.g. 1"-width threads seen by VAULT, ${ }^{5}$ and the contribution from the noise (i.e. photon-noise+read-out noise of the CCD) was confirmed to be below the power at the Nyquist frequency. Both noises were calculated using the same sub-regions as for the calculation of the power spectrum: the photon noise was randomly generated based on the intensity of each corresponding slit-jaw image and the read-out noise was calculated using the dark current images. Hence, the limiting frequency at 2.5" can be considered as an estimation for the achieved resolution, which is in-line with the expected spatial resolution of the slit-jaw optics.

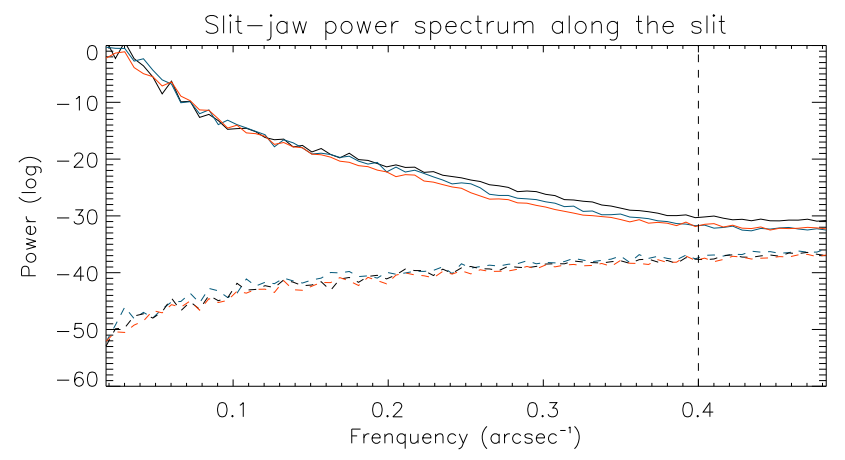

Figure 5. Power spectrum for the slit-jaw images, calculated (integrated radially) in $160 \times 160$ pixels boxes selected at the top (blue solid line), middle (black solid line) and bottom (red solid line) of the slit, all normalized by the first value of the power spectrum at the middle of the slit. The power spectrum of the noise (photon noise+read-out noise) for the three sub-regions is shown in the corresponding dash lines, also normalized similarly as described above. Vertical dash line at 2.5" shows where the power spectrum reach the same power as for the Nyquist frequency (i.e. limiting frequency, approximated as the limit of resolution). 
Figure 6 shows the power spectrums for typical slit image (i.e. spatial) observed in both the channels at the disc center. Because of the limited sample (i.e. only 360 pixels along the slit), analysing particular region along the slit was inconclusive and almost the entire slit had to be used instead (excluding the very edge). Following the same argumentation as for the slit-jaw spatial resolution, the spatial resolution achieved by the spectrograph for both the channels was estimated around 2.9", which is consistent with the results from the pre-flight optical alignment.

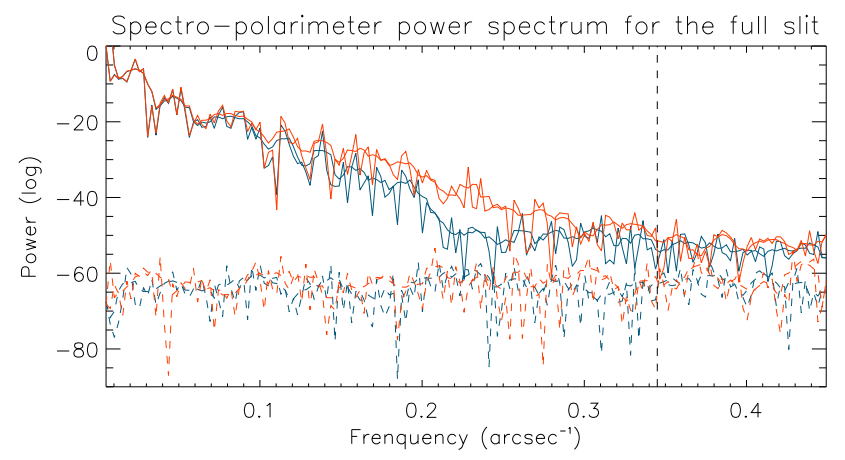

Figure 6. Power spectrums for the spectrograph (channel 1 in blue solid line, channel 2 in red solid line) calculated for almost the full slit length (350 pixels) in the spatial direction, normalized by the first value of the power spectrum for each channel. Thick lines show the smoothed power spectrums. The power spectrum of the noise (photon noise+read-out noise) is shown in dash lines, each normalized using the same value as for the corresponding power spectrum. Vertical dash line at 2.9" shows where the power spectrum reach around the same power as for the Nyquist frequency (i.e. limit of resolution).

Finally, the spectral resolution was estimated by comparing the geocoronal absorption observed by CLASP at the Ly- $\alpha$ line-center with previous spectroscopic observation from the LPSP instrument onboard the OSO8 spacecraft. ${ }^{10}$ These spectral observations also recorded the geocoronal absorption, but with a higher spectral resolution of $0.0020 \mathrm{~nm}$. The LPSP/OSO8 profile was deteriorated using a Gaussian function convolution with increasing FWHM and the geocoronal absorption was compared to the observation by CLASP, at the top, middle and bottom of the slit and for both the channels using a simple rank correlation function. An example of these profiles is shown in Figure 7 for the middle of the slit, and results indicated a spectral resolution of $0.010 \mathrm{~nm}$ at the top, $0.009 \mathrm{~nm}$ at the middle and $0.011 \mathrm{~nm}$ at the bottom of the slit for both the channels. These results are essentially consistent with the required spectral resolution and with the expectation from the pre-flight experiments.
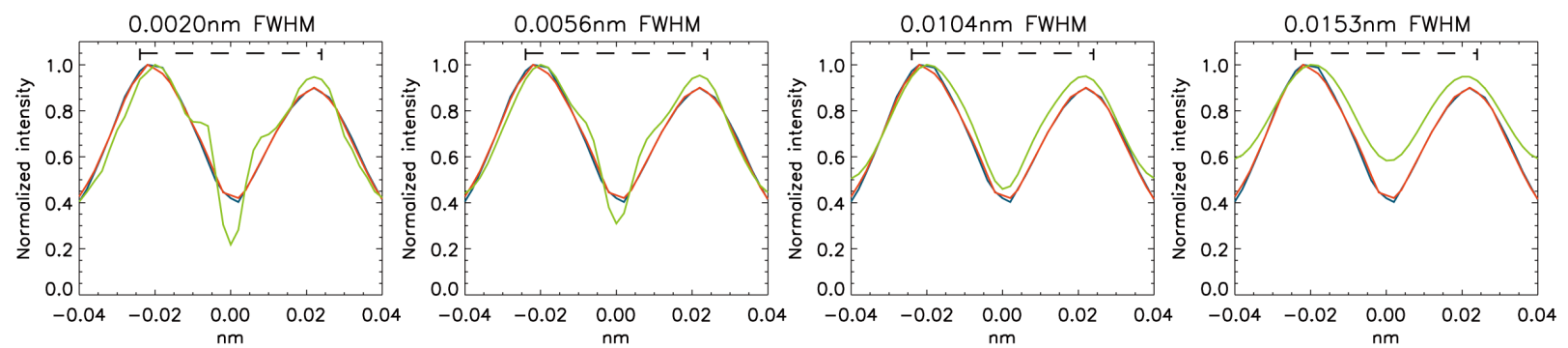

Figure 7. Example of Ly- $\alpha$ spectrum for channel 1 (blue) and channel 2 (red) taken at the center of the slit, compared with spectrum (green) recorded by the OSO8/LPSP instrument in 1975, with a spectral resolution of $0.0020 \mathrm{~nm}$ (left). This profile was deteriorated by a Gaussian function convolution with increasing FWHM and the central geocoronal absorption was compared with the CLASP profiles (only between the two peaks, as shown by the horizontal dash line.) 


\section{CONCLUSION}

The optical alignment of the CLASP sounding rocket instrument was successfully conducted and its performances reached its scientific requirements. Aligning the instrument at Lyman- $\alpha$ raised technical difficulties, as the experiments had to be conducted under vacuum. Hence, experimental methods were designed to pass-by most of these issues. The telescope and spectrograph parts of the instrument were aligned separately:

- For the telescope, the alignment was conducted with a visible-light laser interferometer. Extensive measurements were performed to derive the wavefront error due to the almost transparency of the primary mirror coating in the visible range (3.5\% reflectivity, less than $0.1 \%$ in double-pass).

- For the spectrograph, a custom-made alignment grating with same specifications as the flight Ly- $\alpha$ grating but with a ruling density selected to provide the same diffraction angle at the He-Ne wavelength as the flight grating at Ly- $\alpha$ was used to align the off-axis parabolic mirrors in visible-light. Then, this alignment grating was replaced by the flight grating, and only its Z-tilt, as well as the CCD cameras focus position, were aligned under vacuum using a deuterium lamp and motorized adjustment mechanisms.

Both parts of the instrument met their requirements in term of image quality, and the payload was successfully launched on September $3^{\text {rd }}$ 2015. The flight observations were analysed to determine the achieved spatial and spectral resolutions, which were consistent with the expectations from the pre-flight alignment and with the scientific requirement of the instrument. In conclusion, we presented the results of the methods developed to conduct the optical alignment of the CLASP instrument which ensured its spatial and spectral resolutions, both pre-flight and in-flight. We also hope that the procedures and methods presented in this article would be used for the development of future instruments in the VUV range.

\section{ACKNOWLEDGMENTS}

The authors acknowledge the Chromospheric Lyman-Alpha Spectropolarimeter (CLASP) team. The team was an international partnership between NASA Marshall Space Flight Center, National Astronomical Observatory of Japan (NAOJ), Japan Aerospace Exploration Agency (JAXA), Instituto de Astrofsica de Canarias (IAC) and Institut d'Astro -physique Spatiale ; additional partners include Astronomical Institute ASCR, Lockheed Martin and University of Oslo. Japanese participation is funded by the basic research program of ISAS/JAXA, internal research funding of NAOJ, and JSPS KAKENHI Grant Numbers 23340052, 24740134, 24340040, and 25220703. US participation is funded by NASA Low Cost Access to Space (Award Number 12-SHP 12/2-0283). Spanish participation is funded by the Ministry of Economy and Competitiveness through project AYA201018029 (Solar Magnetism and Astrophysical Spectropolarimetry). French hardware participation was funded by Centre National d'Etudes Spatiales (CNES).

\section{REFERENCES}

[1] Kano, R. et al., "Chromospheric Lyman-Alpha Spectro-Polarimeter (CLASP)," Proc. of SPIE 8443 (2012).

[2] Kobayashi, K. et al., "The Chromospheric Lyman-Alpha Spectropolarimeter: CLASP," Astron. Soc. Pacific 233 (2012).

[3] Trujillo Bueno, J., Štěpán J., J., and Casini, R., "The hanle effect of the hydrogen ly $\alpha$ line for probing the magnetism of the solar transition region," Astrophys. J. 738:L11 (2011).

[4] Belluzzi, L., Trujillo Bueno, J., and Štěpán, J., "The scattering polarization of the ly- $\alpha$ lines of h I and he II taking into account prd and $j$-state interference effects," Astrophys. J. 755:L2 (2012).

[5] Vourlidas, A. et al., "The Structure and Dynamics of the Upper Chromosphere and Lower Transition Region as Revealed by the Subarcsecond VAULT observations," Solar Physics 216, 53-75 (2010).

[6] Giono, G. et al., "Current progress of optical alignment procedure of CLASP's Lyman-alpha polarimetry instrument.," Proc. of SPIE 9144 (2014).

[7] Narukage, N. et al., "Vacuum ultraviolet spectropolarimeter design for precise polarization measurements," Applied Optics 54(8) (2015). 
[8] Suematsu, Y. et al., "The Solar Optical Telescope of Solar-B (Hinode): The Optical Telescope Assembly," Solar Physics 249, 197-220 (2008).

[9] Takeda, M., Ina, H., and Kobayashi, S., "Fourier-transform method of fringe-pattern analysis for computerbased topography and interferometry," Optical Society of America 72(1) (1982).

[10] Gouttebroz, P., Lemaire, P., Vial, J. C., and Arzner, G., "The solar hydrogen Lyman-beta and Lyman-alpha lines Disk center observations from OSO 8 compared with theoretical profiles.," Astrophysical Journal 225, 665-664 (1978). 